DARWIN developed his theory of sexual selection to account for the extravagant secondary sex characters possessed by males of many species. Peacock tails, deer antlers, and bird of paradise plumes are classic examples of characters which hamper survival, but which may increase mating success. Darwin's theory was neatly formalised by R. A. Fisher 36 years ago in his book The Genetical Theory of Natural Selection and later developed as a genetic model by O'Donald (Heredity, 17, $541 ; 1962)$ : briefly, the argument goes like this. Females on the whole tend to be fussier in their choice of mates than are males (the female has more to loose if she makes a bad choice because eggs are more expensive than sperm), so that males end up vying with one another for the attention of a female. Females will always try to choose partners of 'good quality'those which make good parents, or which have good genes to pass on to their children-and in the course of evolution females will develop a preference for external indicators of these qualities. Now suppose that females have begun to evolve a liking for males with green heads, an indicator of good quality: immediately a new selective force comes into play. Males with green heads will now be at an advantage because they are popular with females, and therefore females which choose green-headed males will have sexually attractive sons (assuming inheritance of the green colour), regardless of the quality of these sons in any other respect. The female preference itself generates a selective advantage for green heads, which in turn favours a stronger female preference. It is this positive feedback loop which has

\section{Interferon deglycosylated \\ from David Metz}

INTERFERON is the cellular protein, synthesised by virus-infected animal cells, which effects the protection of other as yet uninfected cells against subsequent virus infection. Interferon has long been believed to be a glycoprotein. The carbohydrate substitution is to be expected of a secreted protein though the experimental evidence for its occurrence has hitherto been somewhat indirect. The principal impediment to the full biochemical characterisation of the various molecular species of interferon has been the difficulty of purifying the material to demonstrable homogeneity. The reason for this difficulty lies in the very high specific activity of interferon which means that

\title{
Sexual selection and the handicap principle
}

from John Krebs

led to the extraordinary elaboration of many male sex characters.

Recently, Zahavi (J. theor. Biol. 53, 205-214; 1975) has come up with an audacious alternative theory of sexual selection. He argues that the females' taste for males with costly, survival-reducing adornments evolves precisely because these features are a handicap. A male which has survived to breeding age in spite of dragging around a huge tail since birth has overtly demonstrated his superior fitness, just as if two athletes ran a mile in four minutes, but one carried a sack of coal, we would conclude that this individual was stronger than his rival. The handicap principle, as Zahavi calls it, has an immediate intuitive appeal. A female benefits by preferring the most handicapped male, because he will pass on good genes to her children, and the handicapped male benefits because the heavy cost of his adornments prevents easy copying by other males. Unfortunately, however, the handicap principle does not work. Mavnard Smith (J. theor. Biol., 57. 345-354; 1976) and Davis and O'Donald ( $J$. theor. Biol., 57. 239-242: 1976) have both analysed Zahavi's idea in more detail, and they show (as many people had suspected) that the verbal argument has serious flaws when translated into strict genetic terms.

Both critiques point to an obvious problem. Even if a handicapped male passes on good genes to his children, he unfortunately also passes on his handicap, which must, according to Zahavi's hypothesis, reduce their survival chances. Perhaps the idea can be salvaged by assuming that the good genes are passed to male and female offspring, but the handicap only goes to the male? Davis and O'Donald point to two difficulties with this argument. First, it is unlikely that genes which are good for a highly adorned male will also be optimal for a cryptic female, and second even if one assumed that the same genes were optimal for both sexes, selection would soon bring females to near their optimum genotype, at which point the advantage of choosing a handicapped male would fade away.

Maynard Smith puts Zahavi's verbal argument into the form of a simulation model, using three loci, one for fitness (high and low), one for a handicap such as tail length, and one for female choosiness-the choosy allele only mates with handicapped males. The fitness of a "high fitness-no handicap' male is set at 1 , and the other fitnesses are calculated bv subtracting a cost of the long tail, and a cost of the 'low fitness' allele. In order to eliminate the effect of female preference (Fisher's idea) the simulation was started with a very low frequency of the 'choosy' allele, the other loci being represented by equal frequencies of their two alleles. The results of the simulation were unambiguous: choosy females and handicapped males declined in frequency, and this was true for a wide range of fitness values. The handicap itself does not favour the evolution of elaborate male sex displays. large volumes of crude preparations (necessarily from tissue culture) yield tiny amounts of pure interferon.

Progress in purifying interferon has been steady if unspectacular in recent years. The current state of the art is well illustrated by two recent papers from Knight on the purification and characterisation of mouse and human interferons. Using basically similar purification procedures, both mouse $\mathrm{L}$ cell interferon and human diploid fibroblast interferon were purified to specific activities of $2 \times 10^{8}$ units per mg protein. The human interferon preparation, when characterised by polyacrylamide gel electrophoresis, displayed a single peak containing essentially all the interferon activity, protein staining capacity and carbohydrate staining capacity of the preparation (Knight, Proc. natn. Acad. Sci. U.S.A., 73, 520; 1976). This preparation, containing a single polypeptide of apparent molecular weight 20,000 , would seem to be the first homogeneous preparation of human interferon.

In contrast, the mouse interferon yielded some dozen discrete bands of activity, following polyacrylamide gel electrophoresis, spanning an apparent molecular weight range of 20,000 to 32,000 . About half of these components were demonstrated to contain carbohydrates, as judged by the periodic acid-Schiff base staining procedure (Knight, J. biol. Chem., 250, 4139; 1975). The reason for this heterogeneity of mouse interferon is as yet undetermined. Variation in the carbohydrate content, as opposed to the primary amino acid sequence, is clearly one possibility.

A second type of heterogeneity in which a variable degree of glycosylation appears to be implicated is the 